\title{
His Second Student
}

\author{
Juris BORZOVS \\ University of Latvia, Faculty of Computing \\ Raiņa bulvāris 19, LV-1586 Rīga, Latvija \\ juris.borzovselu. lv
}

\begin{abstract}
The author claims to be the second graduate student of Rūsiņš Mārtiņš Freivalds. He shares some memories of that time and presents some results in reducibility theory that were not published in peer-reviewed journals before.
\end{abstract}

Keywords: memories on Rūsiṇš Mārtins Freivalds, holographic sets, introreducible sets, autoreducible sets.

\section{In Bygone Days}

It is 1972. Latvia is a part of the USSR, and comrade Brezhnev is at the helm of the country. Another academic year has just started at the Faculty of Physics and Mathematics of the Pêteris Stučka State University of Latvia awarded with the Order of the Red Banner of Labour. The fourth year students majoring in mathematics are provided the option to freely pick four subjects out of six. As part of the four optional classes I decided to choose "Theory of Algorithms" and "Theory of Reducibility". I did not have a slightest clue of the contents of these classes, but at least a lecturer was announced for the former - Jānis Bārzdiņš who had already given us lectures on higher algebra, substituting Docent Vilnis Detlovs who had fallen ill. Detlovs was considered to be a much better lecturer, while Bārzdiņš was supposed to present us with something wildly modern. Meanwhile, the information about the other optional class was scarce; all we knew was its title and a previously unheard lecturer Freivalds. Either of these meant us nothing but we remained open, since we were supposed to be able to change our initial choice after a few first lectures.

So there we sat in the lecture-room waiting for Jānis Bārzdinšs to lead the journey into the theory of algorithms. But instead, a previously unseen young man of average height and rather fragile body structure enters the room, at first sight reminding someone from the Promised Land, of which there are many at the faculty (soon, however, we found out that Freivald's full "identifier” was Rūsinsš Mārtinšs, son of Visvaldis, purely Latvian names, 
so I was totally wrong about him being from the Promised Land), and announces in a perfect Latvian language that docent Bārzdinš has left on a business trip and that he is going to teach us the theory of algorithms for the whole September, and besides - twice as often than specified in the lecture schedule because the basics of the theory of algorithms will be required for his own class. The new lecturer then goes on specifying the time slots when according to him the additional classes will be held. I take a look in my schedule and call out abruptly: "No way! We have some guy named Freivalds scheduled for these hours". The new lecturer then bows and says: "I am Freivalds". The entire auditorium goes into laughter, but I stand totally ashamed.

These days, students at the Faculty of Computing of the University of Latvia may themselves arrange supervisors of their Bachelor's or Master's papers, but back then a supervisor was appointed "from above". I don't know whether Freivalds had any role in that, but I turned out to be under his supervision and in two years I managed to draft the term paper and the graduation paper in the already mentioned topic of the theory of reducibility. It was an invaluable experience. I was the second who developed a graduation paper under the supervision of Freivalds. For two years, Freivalds worked with me individually spending together one hour every week. Moreover, he told me to come even if I failed to make any progress during the week. I doubt whether Professor Freivalds could afford anything like that in the past decades when he already had dozens of students under his supervision.

However banal it might sound, but once following a month of battling the most difficult theorem, I actually found the proof in a dream at night. Fortunately, I could still remember it after waking up.

But the feeling I had following the discovery of the proof in a dream was that my mind had wandered on the other side from the border of my natural abilities, and I am lucky that the mind decided to return home. I decided to never risk like that again, while realizing that the theoretical computer science is not my cup of tea.

The summary of my graduation paper excluding the proof part was published in the collection of the University's scientific papers, however, the results have never appeared in any peer-review magazine or been published in English. It was virtually impossible at that time. Therefore, I attach the results here. Experts will have the opportunity to assess the level of complexity of the theorems Freivald's students have been able to prove over the past decades.

Later, already as a colleague of Professor Freivalds, I watched how he supported his students and cared about them as if they were young kittens. He didn't necessarily look after the most talented or hard-working ones, and constantly encouraged them to publish their first results and took them along to international conferences. If the foreign funds were insufficient to cover fellow-traveling of some students, Freivalds went to the 
Dean's office and gave him his innocent look with the round, sparkling eyes until the latter usually could not resist and found the money after all.

When planning his trips, Freivalds constantly sticked with the airlines and hotel chains he had chosen himself thus collecting bonus points from both. For an outsider, his travel patterns sometimes could seem totally irrational. For instance, a flight from Riga to Japan could initially start in an entirely opposite direction, namely, to Paris with a transfer in Prague, just because Freivalds was collecting bonus points from Czech Airlines and Air France, as well as from Hilton hotels. The logical alternative - a Finnair flight through Helsinki - did not suite, since Finnair was not on the Freivald's list of favourites. Ultimately, however, it was impossible to get angry at him, one could only give a smile for another of his artifices.

Rūsinšs Mārtiņš Freivalds passed away in few seconds while happily planning future works. That was a kind of The Lord's generosity to the great man whose heart in full was given to thousands of people. I will ever feel a small part of his heart in mine.

\section{Holographic Sets}

\section{Yu. V. Borzov}

\section{(Translation from Russian)}

Holograms have an unusual property: an isolated part of a hologram displays the very same image as the whole plate. R.V. Freivald suggested studying a class of natural numbers sets that have similar properties. This paper presents the concept of holographicity and studies its connection with introreducibility ( Jockusch, 1968) and autoreducibility (Trahténbrot, 1970). The mentioned concepts formalize the intuitive notion of mutual dependence (mutual independence) of separate tasks in a mass problem.

Let characteristic function of set $A$ be $f_{A}(x)$. By a sequence we shall mean a partially defined mapping from $\mathrm{N}$ into $\{0,1\}$. If $\mathrm{A}$ is a set, $\{\mathrm{e}\}^{\mathrm{A}}(\mathrm{x})$ is the e-th function partially recursive in $\mathrm{A}$. $\mathrm{W}_{\mathrm{e}}^{\mathrm{A}}$ denotes the domain of $\{\mathrm{e}\}^{\mathrm{A}}(\mathrm{x})$. If $\{\mathrm{e}\}^{\mathrm{A}}(\mathrm{x})=\mathrm{y}$, then this equality follows from a finite number of values of $\mathrm{f}^{\mathrm{A}}(\mathrm{n})$. As it is well known, the function $\{\mathrm{e}\}^{\mathrm{A}}(\mathrm{x})$ is calculated by a Turing machine with an oracle, which can be addressed with the following inquiry: $\mathrm{n} ? € \mathrm{~A}$. The oracle returns 1 if $\mathrm{n} \in \mathrm{A}$, and 0 if $\mathrm{n} \bar{\epsilon} \mathrm{A}$. If $\sigma$ is a sequence, $\mathrm{A}$ is a set and for each $\mathrm{n}$ in the domain of $\sigma, \sigma(\mathrm{n})=\mathrm{f}^{\mathrm{A}}(\mathrm{n})$, then $\{\mathrm{e}\}^{\sigma}(\mathrm{x})$ is the e-th function partially recursive in $\sigma$, i.e. this function is calculated by Turing machine with an oracle, which can be addressed with the following inquiry: $\mathrm{n} \epsilon$ ? $\mathrm{A}$; the oracle returns 1 
if $\sigma(n)=1$, returns 0 if $\sigma(n)=0$ and 2 (empty answer), if $\sigma(n)$ is not defined. $W_{e}{ }^{\sigma}$ denotes the domain of $\{\mathrm{e}\}^{\sigma}(\mathrm{x})$. If $\{\mathrm{e}\}^{\sigma}(\mathrm{x})=\mathrm{y}$, then this equality follows from finitely many values of $\sigma(\mathrm{n})$. If sequence $\sigma$ is a total function then A-partial-recursiveness (A-recursiveness) is equal to 6 -partial-recursiveness (б-recursiveness).

$\{e\}^{\sigma}=A$ if and only if for each $x \in N\{e\}^{\sigma}(x)=f_{A}(x) \cdot W_{e}^{\sigma}=A$ if and only if for each $x \in A$ $\{e\}^{\sigma}(x)=f_{A}(x)$.

DEFINITION 1. A set A is called holographic, if for each sequence $\sigma$ that satisfies the following conditions:

a) an infinite amount of $\mathrm{n}$ exists, for which $\sigma(\mathrm{n})=1$;

b) an infinite amount of $\mathrm{n}$ exists, for which $\sigma(\mathrm{n})=0$;

c) for each $\mathrm{n}$ in the domain of $\sigma, \sigma(n)=f^{A}(n)$;

there exists a number $\mathrm{e}$, for which $\{\mathrm{e}\}^{\sigma}=\mathrm{A}$.

DEFINITION 2. A set A is called uniformly holographic, if there is a number e such that $\{\mathrm{e}\}^{\sigma}=\mathrm{A}$ for each sequence $\sigma$ that satisfies the conditions a), b), c).

Let us explain the definitions with the help of the following interpretation. Given: a set A and an infinite tape with slots; each slot contains a natural number and the notation about its belonging to A. Numbers are placed on the tape in an ascending order. The tape is covered by a black slotted tape that allows to see only some of the numbers on the tape below it. No information is given about numbers covered by the black tape. For example,

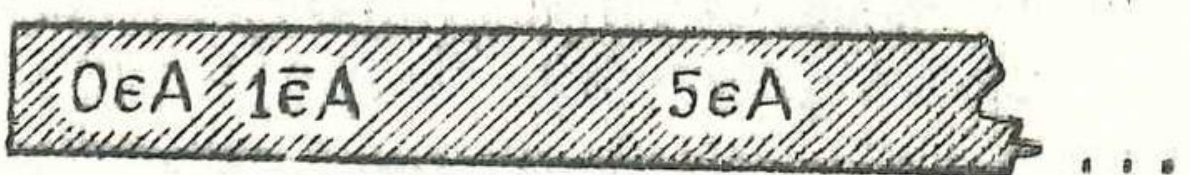

Thus the black slotted tape is an oracle that provides partial information about belonging of some numbers to $\mathrm{A}$.

Definition 1 may be reformulated as follows: a set A is holographic provided that for each black tape with infinitely many slots with numbers that belong to A, and infinitely many slots with numbers that do not belong to $\mathrm{A}$, there is an algorithm that can solve the problem of belonging of any given natural number to $\mathrm{A}$.

Definition 2 requires the existence of a single algorithm for all such black tapes. 
DEFINITION 3. (Jockusch, 1968). Introreducible and uniformly introreducible sets are defined by using the definitions 1 and 2 respectively, with the condition b) put aside.

In our interpretation the problem of occurrence for these sets can be solved by using black tapes uncovering infinitely many slots with numbers that belong to A.

According to these definitions, each (uniformly) introreducible set and each (uniformly) co-introreducible set is (uniformly) holographic. It is also easy to show that there exists a continuum of holographic sets (as also a continuum of non-holographic sets).

DEFINITION 4. (Trakhténbrot, 1970). A set A is called autoreducible if there is an e such that $\{\mathrm{e}\}^{\sigma}=\mathrm{A}$, where for each $\mathrm{x}_{\mathrm{o}} \in \mathrm{N}$ in the calculation of $\{\mathrm{e}\}^{\sigma}\left(\mathrm{x}_{0}\right)$ the sequence $\sigma$ is undefined only at $\mathrm{x}_{0}$.

In our interpretation this means - the black tape covers only the number whose belonging to $\mathrm{A}$ is being calculated at the moment.

LEMMA 1. There exists a continuum of autoreducible sets which are not holographic.

THEOREM 1. There exists holographic, but not uniformly holographic set.

LEMMA 2. There exists a non-holographic recursively enumerable set.

LEMMA 3. If a set $\mathrm{A}$ is (uniformly) holographic, then $\overline{\mathrm{A}}$ is also (uniformly) holographic.

LEMMA 4. There exist uniformly holographic sets $A_{i}$ and $B_{i}$ such that $A_{1} \cup B_{1}, A_{2} \cap B_{2}$, $\mathrm{A}_{3} \backslash \mathrm{B}_{3}, \mathrm{~A}_{4} \mathrm{joinB}_{4},\left(\mathrm{~A}_{5} \cap \bar{B}_{5}\right) \mathrm{U}\left(\overline{\mathrm{A}}_{5} \cap \mathrm{B}_{5}\right)$ are not holographic.

LEMMA 5. There exist Turing equivalent uniformly holographic sets A and B such that AUB is not uniformly holographic.

DEFINITION 5. A set A is called holographic enumerable if for each sequence $\sigma$, that satisfies conditions a), b), c), there is an e such that $\mathrm{W}_{\mathrm{e}}{ }^{6}=\mathrm{A}$. Let us call the set uniformly holographic enumerable, if there exists such an e for every such 6.

THEOREM 2. If set A is uniformly holographic enumerable and introreducible, then it is also uniformly holographic. At the same time it is not always uniformly introreducible. 
THEOREM 3. Every holographic set is autoreducible.

LEMMA 6. If $\mathrm{A}$ and $\overline{\mathrm{A}}$ are (uniformly) holographic enumerable, then $\mathrm{A}$ is (uniformly) holographic.

THEOREM 4. There exists a uniformly holographic set which is neither introreducible nor co-introreducible.

Thus we have established that holographic sets represent a natural intermediate link between introreducible and autoreducible sets.

It should be noted that if considering infinite sequences $\sigma$ without imposing conditions a) and b), in case of uniformity we will get exactly the class of recursive sets.

\section{References}

Jockusch Jr., C. G. (1968). Uniformly introreducible sets. Journal of Symbolic Logic, vol. 33, No. 4, pp. $521-536$.

Trakhténbrot, B. A. (1970). Ob avtosvodimosti. Doklady Akadémii Nauk SSSR, vol. 192, pp.

1224-1227. Trakhtenbrot B. A. On autoreducibility. English translation of the preceding by M. Machover. Soviet mathematics, vol. 11, no. 3, pp. 814-817.

Received September 13, 2016, accepted October 25, 2016 